Portland State University

PDXScholar

Electrical and Computer Engineering Faculty

Publications and Presentations

$12-31-1983$

\title{
Lateral Coherence of Aerosol-Scattered Laser Radiation
}

\author{
Donald D. Duncan \\ Portland State University \\ D. E. Larch
}

Follow this and additional works at: https://pdxscholar.library.pdx.edu/ece_fac

Part of the Electrical and Computer Engineering Commons

Let us know how access to this document benefits you.

\section{Citation Details}

"Lateral Coherence of Aerosol-Scattered Laser Radiation," D. D. Duncan and D. Larch, Optics Letters, V. 9, pp. 1-3 (1984).

This Article is brought to you for free and open access. It has been accepted for inclusion in Electrical and Computer Engineering Faculty Publications and Presentations by an authorized administrator of PDXScholar. Please contact us if we can make this document more accessible: pdxscholar@pdx.edu. 


\title{
Lateral coherence of aerosol-scattered laser radiation
}

\author{
D. D. Duncan* \\ Pacific-Sierra Research Corporation, 1401 Wilson Boulevard, Arlington, Virginia 22209 \\ D. E. Larch \\ Pacific-Sierra Research Corporation, 3138 Presidential Drive, Fairborn, Ohio 45324
}

Received May 12, 1983; revised manuscript received September 29, 1983

\begin{abstract}
To assess the probability of detection (by interferometric techniques) of illumination by a coherent source, we characterize the lateral coherence of the off-axis aerosol scattered radiation by applying the van Cittert-Zernike theorem. We find that, for $1.061-\mu \mathrm{m}$ radiation at a target range of $1 \mathrm{~km}$, the scattered radiation beyond $3.5 \mathrm{~cm}$ off axis does not display appreciable lateral coherence.
\end{abstract}

A sensor that discriminates between coherent and incoherent radiation is of use in detecting illumination that is due to a laser. In such systems, it is desirable to detect near misses as well as direct illumination of the target by the designator. The mechanisms by which a near miss may be detected is aerosol forward scatter out of the beam onto the target.

Because of the aerosol scattering, the off-axis radiation (in many cases of interest) possesses insufficient lateral coherence for a sensor to perceive the incident flux as having originated from a (spectrally) coherent source, i.e., a laser. It is assumed that the field decorrelation effects that are due to turbulence are minor compared with those that are due to aerosol scatter. Correlation lengths for propagation through turbulence are of the order of centimeters ${ }^{1,2}$ for the geometrical configuration under consideration. Correlation lengths for aerosol scatter are much shorter.

Of course, lateral coherence is a necessary but not sufficient condition for detection. For example, the flux density incident upon the detector must be above the system noise level. In this Letter we quantify the conditions under which the forward-scattered radiation displays appreciable lateral coherence. We do not address other conditions necessary for detection.

Van de Hulst ${ }^{3}$ has demonstrated that the light scattered by an aerosol medium exhibits no lateral coherence. Nevertheless, Born and Wolf, ${ }^{4}$ using the van Cittert-Zernike theorem, have shown that under certain geometric configurations an extended thermal source (i.e., an assemblage of mutually incoherent point radiators) may display a degree of lateral coherence. It is along the lines of Born and Wolf's analysis that we quantify the transverse coherence of the forward-scattered radiation.

With analogy to the Huygens-Fresnel theorem, ${ }^{4}$ we assume that the electromagnetic field at point $(x, y, L)$ can be written as the superposition of the fields that is due to point sources (the scatter from aerosol particles) lying along the $z$ axis. See Fig. 1. This is expressed conceptually as

$$
E\left(x_{1}, y_{1}, L\right)=\int_{0}^{L} \mathrm{~d} z_{1} e\left(\mathbf{r}_{1}, z_{1}\right) \frac{\exp \left[i k\left(z_{1}+R_{1}\right)\right]}{R_{1}},
$$

where $e$ is the apparent source distribution. The explicit dependence of the source on the observation point simply allows for nonisotropic elemental radiators. The complex correlation of the fields in the $z=L$ plane then is given by

$$
\begin{aligned}
J\left(\mathbf{r}_{1}, \mathbf{r}_{2}\right) & =\left\langle\int \mathrm{d} z_{1} e\left(\mathbf{r}_{1}, z_{1}\right) \frac{\exp \left[i k\left(z_{1}+R_{1}\right)\right]}{R_{1}}\right. \\
& \left.\times \int \mathrm{d} z_{2} e^{*}\left(\mathbf{r}_{2}, z_{2}\right) \frac{\exp \left[-i k\left(z_{2}+R_{2}\right)\right]}{R_{2}}\right\rangle,
\end{aligned}
$$

where the angle brackets denote ensemble averaging. Equation (2) can be rewritten as

$$
\begin{aligned}
& J\left(\mathbf{r}_{1}, \mathbf{r}_{2}\right)=\int \mathrm{d} z_{1} \int \mathrm{d} z_{2} \frac{\exp \left[i k\left(z_{1}-z_{2}+R_{1}-R_{2}\right)\right]}{R_{1} R_{2}} \\
& \times\left\langle e\left(\mathbf{r}_{1}, z_{1}\right) e^{*}\left(\mathbf{r}_{2}, z_{2}\right)\right\rangle
\end{aligned}
$$

The term in angle brackets is simply the correlation of the fields that is due to the two point sources (i.e., scatterers) at $z=z_{1}$ and $z=z_{2}$. Without loss of gen-

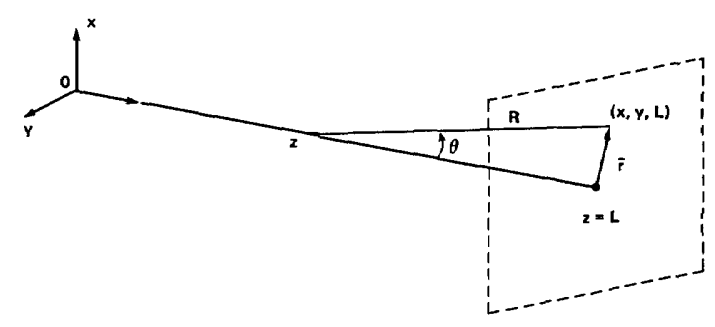

Fig. 1. Illustration of scenario. 
erality, the scatterers are assumed to be independent so that we have

$$
\begin{aligned}
\left\langle e\left(\mathbf{r}_{1}, z_{1}\right) e^{*}\left(\mathbf{r}_{2}, z_{2}\right)\right\rangle & \\
& =P_{0} \beta_{\mathrm{se}} P(\theta) \delta\left(z_{1}-z_{2}\right) T(0, z) T(z, \mathbf{r}),
\end{aligned}
$$

where $P_{0}$ is the laser output power, $\beta_{\mathrm{sc}}$ is the aerosol scattering cross section per unit volume, $P(\theta)$ is the aerosol single-scatter phase function, and $T(0, z)$ and $T(z, \mathbf{r})$ are transmission between source and scatter point and thence to the field point.

In Eq. (4), the angle $\theta$ represents an average scatter angle. (We are interested in a small region in the $z=$ $L$ plane.) Also, the point $\mathbf{r}$ denoted explicitly in the (slowly varying) transmission function represents an average position. This equation expresses the apparent radiance [at point $(\mathbf{r}, L)$ in the detector plane] per unit length [at point $(0, z)]$ of the laser beam. See, for example, Ref. 5 .

We now define the complex degree of coherence as

$$
\mu\left(\mathbf{r}_{1}, \mathbf{r}_{2}\right)=\frac{J\left(\mathbf{r}_{1}, \mathbf{r}_{2}\right)}{\left[I\left(\mathbf{r}_{1}\right)\right]^{1 / 2}\left[I\left(\mathbf{r}_{2}\right)\right]^{1 / 2}},
$$

where

$$
\begin{aligned}
I\left(\mathbf{r}_{1}\right) \simeq & I\left(\mathbf{r}_{2}\right) \\
& =P_{0} \beta_{\mathrm{sc}} \int_{0}^{L} \mathrm{~d} z P(\theta) T(0, z) T(z, \mathbf{r}) / R^{2} .
\end{aligned}
$$

By employing the notation

$$
\sigma=\frac{\mathbf{r}_{1}+\mathbf{r}_{2}}{2}, \quad \delta=\mathbf{r}_{1}-\mathbf{r}_{2},
$$

combining Eqs. (3)-(6), and performing one of the longitudinal integrals (made trivial by the delta function), we obtain

$\mu(\sigma, \delta)$

$$
=\frac{\int_{0}^{L} \mathrm{~d} z P(\theta) T(0, z) T(z, \sigma) \frac{\exp \left[i k\left(R_{1}-R_{2}\right)\right]}{R_{1} R_{2}}}{\int_{0}^{L} \mathrm{~d} z P(\theta) T(0, z) T(z, \sigma) \frac{1}{R_{1} R_{2}}},
$$

$$
=\frac{\int_{0}^{L} \mathrm{~d} z P(\theta) T(0, z) T(z, \sigma)\left[2 J_{1}(u) / u\right] /(L-z)^{2}}{\int_{0}^{L} \mathrm{~d} z P(\theta) T(0, z) T(z, \sigma) /(L-z)^{2}},
$$

where $J_{1}$ is the Bessel function of the first kind and order unity and $u$ is given by

$$
u=\frac{k D \sigma}{2(L-z)} .
$$

At this point, we digress a bit to point out that, because the detector does not have a hemispherical field of view (FOV), it is blind to off-axis scatter in the vicinity of the plane $z=L$. The implication of this is that, rather than integrate on the interval $(0, L)$, we should integrate on the interval $(0, L-\sigma \tan \alpha)$, where $\alpha$ is the FOV half-angle.

We now assume that the functions $P$ and $T$ are slowly varying with respect to the other terms in the integrands. With this approximation, the integration in the denominator of Eq. (10) can be performed to yield

$$
\begin{aligned}
\mu(\sigma)= & \left(\frac{\frac{\sigma / L}{\tan \alpha}}{1-\frac{\sigma / L}{\tan \alpha}}\right) L \\
& \times \int_{0}^{L-\sigma / \tan \alpha} \mathrm{d} z\left[2 J_{1}(u) / u\right] /(L-z)^{2} .
\end{aligned}
$$

This expression can be further simplified by the change of variable

$$
u \equiv \frac{k D \sigma}{2(L-z)}=x .
$$

Equation (11) then yields

$$
\mu(\sigma)=\left(\frac{\frac{\sigma / L}{\tan \alpha}}{1-\frac{\sigma / L}{\tan \alpha}}\right)\left(\frac{2 L}{k D \sigma}\right) \int_{k \mathrm{D} \sigma / 2 L}^{k D \tan \alpha / 2} \mathrm{~d} x\left[2 J_{1}(x) / x\right] .
$$

$$
\mu(\sigma, \delta)=\frac{\int_{0}^{L} \mathrm{~d} z P(\theta) T(0, z) T(z, r) \exp [i k \sigma \cdot \delta /(L-z)] /(L-z)^{2}}{\int_{0}^{L} \mathrm{~d} z P(\theta) T(0, z) T(z, r) /(L-z)^{2}} .
$$

To obtain a measure of the importance of the lateral coherence, we define a figure of merit as the integral of Eq. (8) over the detector aperture:

$$
\begin{aligned}
\mu(\sigma) \equiv & \frac{1}{A} \int_{A} \mathrm{~d} \delta \mu(\sigma, \delta) \\
& =\left[\pi\left(\frac{D}{2}\right)^{2}\right]^{-1} \int_{0}^{2 \pi} \mathrm{d} \phi \int_{0}^{D / 2} \mathrm{~d} \delta \delta \mu(\sigma, \delta \phi),
\end{aligned}
$$

where the aperture diameter is $D$.

'The angular and radial integrations are easily performed to yield
For many cases of interest, the upper limit on the integration in Eq. (13) may safely be extended to infinity. Further, defining a normalized figure of merit,

$$
\mu_{N}(\sigma)=\mu(\sigma) \mu(0)
$$

we have, finally,

$$
\mu_{N}(\sigma)=\left(1-\frac{\sigma / L}{\tan \alpha}\right)^{-1}\left[1-\int_{0}^{\pi D \sigma / \lambda L} \mathrm{~d} x J_{1}(x) / x\right] .
$$




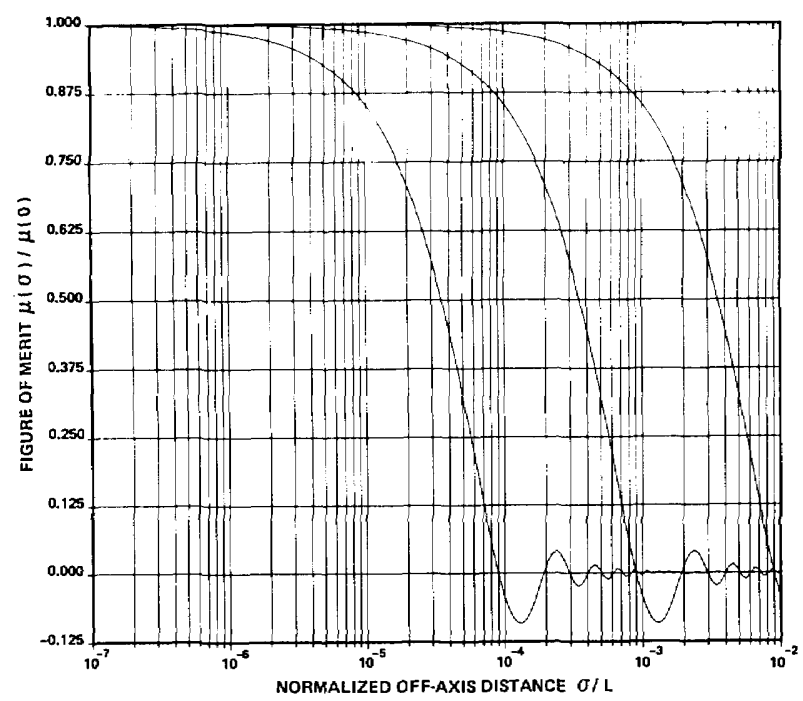

Fig. 2. Lateral coherence figure of merit. The three curves correspond, from left to right, to $D$ equaling $1.0,0.1$, and 0.01 cm.

This expression was evaluated with a compound Gauss-Legendre quadrature method of numerical integration $^{6}$ for the following values of the relevant parameters:

$$
\begin{aligned}
\alpha & =30^{\circ}, \\
\lambda & =1.061 \mu \mathrm{m}, \\
0.01 \mathrm{~cm} & \leq D \leq 1 \mathrm{~cm}, \\
10^{-7} & \leq \frac{\sigma}{L} \leq 10^{-2} .
\end{aligned}
$$

Figure 2 shows the results of this calculation. The ordinate is the figure of merit, and the abscissa is the ratio of the perpendicular distance from the beam axis to the center of the detector, $\sigma$, to the length of the beam axis, $L$.
We arbitrarily choose a value of $1 / 2$ for the normalized figure of merit, beyond which we consider the lateral coherence to be negligible. For example, with a $1-\mathrm{km}$ beam path and a 1-cm detector aperture diameter, the off-axis distance beyond which the scattered flux possesses no appreciable lateral coherence is given by

$$
\sigma / L=3.5 \times 10^{-5} \text {, }
$$

or

$$
\sigma=3.5 \mathrm{~cm} \text {. }
$$

Such a small off-axis distance is of no practical interest.

The implication of these results is of interest in predicting probability of detection versus false-alarm rates for detectors relying on spatial coherence.

This research was supported by the U.S. Air Force under contract no. F33657-82-C-0033.

* Present address, Johns Hopkins University, Applied Physics Laboratory, Johns Hopkins Road, Laurel, Maryland 20707.

\section{References}

1. R. F. Lutomirski and R. G. Buser, "Mutual coherence function of a finite optical beam and application to coherent detection," Appl. Opt. 12, 2153-2160 (1973).

2. R. L. Fante, "Mutual coherence function and frequency spectrum of a laser beam propagating through atmospheric turbulence," J. Opt. Soc. Am. 64, 592-598 (1974).

3. H. C. van de Hulst, Light Scattering by Small Particles (Wiley, New York, 1957).

4. M. Born and E. Wolf, Principles of Optics, 4th ed. (Pergamon, Oxford, 1970).

5. D. Deirmendjian, Electromagnetic Scattering on Spherical Polydispersions, Rand Corp. Rep. R-456-PR (American Elsevier, New York, 1969).

6. M. Abramowitz and I. Stegun, Handbook of Mathematical Functions with Formulas, Graphs, and Mathematical Tables (U.S. Government Printing Office, Washington, D.C., 1964). 